\title{
Expression profiling of microRNAs in human bone tissue from postmenopausal women
}

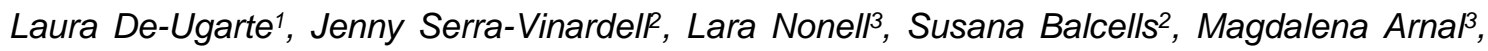
Xavier Nogues ${ }^{1}$, Leonardo Mellibovsky', Daniel Grinberg², Adolfo Diez-Perez ${ }^{1}$, Natalia GarciaGiralt ${ }^{1}$

${ }^{1}$ Musculoskeletal Research Group, IMIM (Hospital del Mar Medical Research Institute), Centro de Investigación Biomédica en Red de Fragilidad y Envejecimiento Saludable (CIBERFES), ISCIII, Barcelona, Spain.

2Department of Genetics, Microbiology and Statistics, Facultat de Biologia, Universitat de Barcelona, IBUB, IRSJD, Centro de Investigación Biomédica en Red de Enfermedades Raras (CIBERER), ISCIII, Barcelona, Spain.

${ }^{3}$ Microarray Analysis Service, IMIM (Hospital del Mar Medical Research Institute), Barcelona, Spain.

Corresponding author:

*Natalia Garcia-Giralt, PhD, IMIM-Hospital del Mar, C/ Dr. Aiguader 88, 08003 Barcelona, SPAIN

E-mail: ngarcia@imim.es

Phone: 00343160497 


\section{Abstract}

Bone tissue is composed of several cell types, which express their own microRNAs (miRNAs) that will play a role in cell function. The set of total miRNAs expressed in all cell types configures the specific signature of the bone tissue in one physiological condition.

The aim of this study was to explore the miRNA expression profile of bone tissue from postmenopausal women. Tissue was obtained from trabecular bone and was analyzed in fresh conditions $(n=6)$. Primary osteoblasts were also obtained from trabecular bone $(n=4)$ and human osteoclasts were obtained from monocyte precursors after in vitro differentiation $(n=5)$. MicroRNA expression profiling was obtained for each sample by microarray and a global miRNA analysis was performed combining the data acquired in all the microarray experiments.

From the 641 miRNAs detected in bone tissue samples, 346 (54\%) were present in osteoblasts and/or osteoclasts. The other $46 \%$ were not identified in any of the bone cells analyzed. Intersection of osteoblast and osteoclast arrays identified 101 miRNAs shared by both cell types, which accounts for $30-40 \%$ of miRNAs detected in these cells. In osteoblasts, 266 miRNAs were detected, of which $243(91 \%)$ were also present in the total bone array, representing $38 \%$ of all bone miRNAs. In osteoclasts, 340 miRNAs were detected, of which 196 $(58 \%)$ were also present in the bone tissue array, representing $31 \%$ of all miRNAs detected in total bone.

These analyses provide an overview of miRNAs expressed in bone tissue, broadening our knowledge in the microRNA field.

Keywords: microRNA, microarrays, bone tissue, osteoblast, osteoclast 


\section{Introduction}

Bone tissue is made up of cells, vessels and a mineralized extracellular matrix. The bone cell population consists of two distinct lineages: the osteogenic lineage (including stromal cells, preosteoblasts, osteoblasts, osteocytes and bone lining cells) and the osteoclastic lineage, represented by osteoclasts. The maintenance of normal bone structure is driven by cell function and the intercellular cross-talk of these bone cells.

Osteoblasts, which derive from mesenchymal stem cells (MSC), are well known for their function in bone formation and account for up to $6 \%$ of total resident cells in the bone. On the other hand, osteoclasts are bone resorptive cells and represent approximately $1-2 \%$ of total bone-resident cells. These terminally differentiated giant multinucleated cells are derived from the fusion of mononuclear progenitors of the monocyte/macrophage hematopoietic lineage [1]. The third main bone cell is the osteocyte, which comprises $90-95 \%$ of all bone cells in adult bone. This terminally differentiated osteoblast becomes surrounded by matrix during the process of bone formation. Osteocytes have a mechanosensory function and orchestrate bone formation and resorption by acting on osteoblast and osteoclast recruitment, depending on different mechanical and metabolic conditions [2] [3].

MicroRNAs (miRNAs) are single-strand non-coding RNAs, about 20-25 nucleotides in length, that post-transcriptionally regulate gene expression mainly by binding to the 3'UTR of their target messenger RNAs (mRNA). Each specific cell type expresses its own miRNAs as needed for the cell function at a precise moment. The set of total miRNAs expressed by all bone cell types at a given physiological point configures the specific signature of the bone tissue at that point.

Up to date, several miRNAs have been reported to regulate the differentiation and activity of osteoblasts and osteoclasts [4] by targeting genes with a key role in bone turnover, while many more remain to be discovered. Identifying the total set of miRNAs expressed in bone and their specific functions should broaden our current knowledge of the action mechanisms of the bone itself and give insights into the causes and possible treatments of skeleton disorders. In this regard, lots of human studies on miRNAs in the bone field are focusing on postmenopausal 
status since it is involved in the bone remodeling alteration leading to the osteoporosis development and an increased risk of bone fractures [5] [6] [7] [8].

This study aims to explore the expression patterns of microRNAs in bone tissue from postmenopausal women by mapping them into the different bone cells. For this analysis, microarrays were hybridized with miRNA samples from of whole bone tissue, osteoblasts (hOBs) and osteoclasts (hOCs). The profiles obtained were then overlaid to develop a global vision of miRNAs present in bone.

\section{Materials and Methods}

Ethics Statement

The Clinical Research Ethics Committee of Parc de Salut MAR approved the present research (Registry number 2010/3882/I and 2013/5266/I). The approved protocols for obtaining fresh bone (and primary osteoblasts) from hip or knee samples otherwise discarded at the time of orthopedic surgery as well as protocols for obtaining blood samples for monocyte isolation were explained to potential participants, who provided written informed consent before being included in the study.

Collection of bone samples

Knee $(n=4)$ and femoral neck trabecular $(n=6)$ bone were obtained from postmenopausal women undergoing knee or hip replacement due to osteoarthritis. Knee bone was used for obtaining hOBs whereas femoral neck was used for whole bone tissue analysis. None of the participants had a history of metabolic or endocrine disease, chronic renal failure, chronic liver disease and malignancy, Paget 's disease of bone, malabsorption syndrome, hormone replacement therapy, anti-resorptive agents, oral corticosteroids, anti-epileptic drugs, or treatment with lithium, heparin, or warfarin.

Primary osteoblast culture

Human primary osteoblasts were obtained from knee trabecular bone. Bony tissue was cut up into small pieces, washed in phosphate buffered solution (PBS, Gibco by Life Technologies; 
Paisley, UK) to remove non-adherent cells, and placed on a $140 \mathrm{~mm}$ culture plate. Samples were incubated in hOB medium: Dulbecco's Modified Eagle Medium (DMEM; Gibco; Invitrogen, Paisley, UK), supplemented with 10\% fetal bovine serum (FBS; Sigma-Aldrich; St. Louis, USA), $100 \mathrm{U} / \mathrm{ml}$ penicillin/streptomycin (Sigma-Aldrich; St. Louis, USA), $0.4 \%$ fungizone (Gibco by Life Technologies; Paisley, UK), and $100 \mu \mathrm{g} / \mathrm{ml}$ ascorbic acid (Sigma-Aldrich; Steinheim, Germany). After approximately 3 weeks of culture and before reaching confluence, cells were processed for RNA extraction. In parallel, alkaline phosphatase (ALP) activity was measured with Abcam's Alkaline Phosphatase Assay Kit (Colorimetric) in order to confirm the osteoblastic phenotype.

\section{Collection of osteoclasts}

Peripheral blood samples were obtained from 5 postmenopausal women without history of metabolic or endocrine disease, chronic renal failure, chronic liver disease, malignancy, Paget's disease of bone, malabsorption syndrome, hormone replacement therapy, anti-osteoporosis agents, oral corticosteroids, anti-epileptic drugs, or treatment with lithium, heparin, or warfarin.

Monocytes, the osteoclast precursors, were isolated from mononuclear cells with the RosetteSep ${ }^{\mathrm{TM}}$ Human Monocyte Enrichment Cocktail (Stemcell technologies, Vancouver, BC, Canada) following the manufacturer's instructions. After isolating the monocyte fraction, the CD14 marker was determined by flow cytometry, obtaining a culture purity of $90 \%$ CD14+ cells. Monocytes were seeded at $5 \times 10^{5}$ cells on $10 \mathrm{~mm}$ glass coverslips (Marienfeld, LaudaKönigshofen, Germany) and incubated for 2 hours at $37^{\circ} \mathrm{C}$ in $5 \% \mathrm{CO}_{2}$ in a completed medium of alpha-MEM (Gibco) supplemented with 10\% FBS (Gibco), 2 mM Glutamax (Gibco), and 1\% penicillin/streptomycin (Gibco). Non-adherent cells were washed out and adherent cells were used for the osteoclast differentiation. The adherent cells were cultured in completed medium supplemented with $25 \mathrm{ng} / \mathrm{ml}$ M-CSF (Peprotech) and $50 \mathrm{ng} / \mathrm{ml}$ RANKL (Peprotech). All the cultures were maintained until the generation of multinucleated giant cells, which occurred at 21-24 days of culture. Culture medium was changed once every 3 days and M-CSF and RANKL were added at each medium change. TRAP and osteoclast actin ring staining were tested to confirm osteoclast differentiation in culture conditions. 


\section{RNA extraction}

For RNA extraction of whole bone tissue, bony fragments were extracted from the transcervical region of the femoral neck. Fresh bone samples were cut into small fragments, triple washed in PBS, and stored at $-80^{\circ} \mathrm{C}$ until use. Total RNA extraction from total bone tissue, osteoblasts and osteoclasts was performed following the manufacturer's instructions for the High Pure RNA Isolation kit (Roche Diagnostics, Indianapolis, USA) and the miRNeasy Mini kit (Qiagen). The quality of the total RNA was verified by an Agilent 2100 Bioanalyzer profile.

MicroRNA array hybridization and data analysis

The microarray procedure for whole bone tissue and osteoblast samples was conducted at Exiqon Services, Denmark, as previously described [5]. Total RNA from both test samples (250 $\mathrm{ng}$ of total bone or $750 \mathrm{ng}$ of primary osteoblasts) and reference samples was labeled with Hy3 ${ }^{\mathrm{TM}}$ and $\mathrm{Hy} 5^{\mathrm{TM}}$ fluorescent dyes, respectively, using the miRCURY LNA ${ }^{\mathrm{TM}}$ microRNA HiPower Labeling Kit, Hy3 $3^{\mathrm{TM}} / \mathrm{Hy} 5^{\mathrm{TM}}$ (Exiqon, Denmark) as indicated by the manufacturer. Values for the miRNA analysis were calculated as log2 transformed normalized median ratios. Criteria for miRNA selection were expression values higher than 1.2 times the 25th percentile of the overall intensity values per array in at least three samples of total bone tissue or in two osteoblast samples.

The osteoclast microarray was performed in the Servei d'Anàlisi de Microarrays of the Hospital del Mar Medical Research Institute using the Affymetrix GeneChip® technologic platform. Samples were poly (A)-tailed and biotin-ligated using the Genisphere FlashTag ${ }^{\mathrm{TM}}$ Biotin HSR RNA Labelling Kit. After sample processing and before hybridization, biotin labeling was confirmed with the Enzyme Linked Oligosorbent Assay (ELOSA). Samples were then hybridized into the GeneChip $\AA$ miRNA 4.0 arrays during 16 hours at $48^{\circ} \mathrm{C}$ and $60 \mathrm{rpm}$ in a GeneChip $\AA$ Hybridization Oven 640. Following hybridization, the arrays were washed and stained in the GeneChip ${ }^{\circledR}$ Fluidics Station 450, using the GeneChip ${ }^{\circledR}$ Hybridization, Wash and Stain kit. The stained arrays were scanned with the GeneChip® Scanner 3000 7G, generating CEL files for each array. Data quality control was assessed using Affymetrix Expression Console software. All arrays met the quality control criteria. 
Normalization was performed with $\mathrm{R}$ (version 3.2.3) using the Robust Multi-array Average

Results

Sample description

The anthropometric features of all patient groups are shown in Table 1. All samples were obtained from postmenopausal women with no history of metabolic bone disorders. All samples are independent from each other without overlapping participants.

Human osteoblast (hOBs) cultures were characterized by means of ALP detection. Primary osteoblasts showed similar ALP levels than cultures of osteosarcoma cell lines confirming the osteoblastic phenotype of cells used for array analysis (Supplemental figure 1).

Human osteoclasts (hOCs) were in vitro differentiated from monocytic progenitors. Mature TRAP-positive giant multinucleated osteoclasts were detected at 21 to 24 days of culture in the presence of M-CSF and RANKL. Functional osteoclasts were confirmed microscopically by the presence of three or more nuclei, the presence of podosomes, and the formation of actin ring (Supplemental figure 2).

\section{Global bone miRNA analysis}

Three microRNA profiles were obtained by array hybridization: one from total fresh bone $(n=6)$, another from hOBs $(n=4)$ and a third from in vitro differentiated hOCs $(n=5)$. Bone and osteoblast arrays were from Exiqon while the osteoclast array was from Affymetrix. Only miRNAs shared between platforms $(n=2057)$ were included in the study. miRNAs expressed at least in $2 / 4$ of the hOBs, $2 / 5$ of the hOCs, and $3 / 6$ of the bone samples were included for the miRNA intersection analysis, resulting in 801 miRNAs (Figure 1).

From the 641 miRNAs detected in bone samples, $345(54 \%)$ were found to be present in osteoblasts and/or osteoclasts. The other $46 \%$ of miRNAs detected in bone $(n=296)$ were not 
identified in any of the bone cells analyzed. This group of miRNAs might be specific to other bone cells such as osteocytes, which are the most prevalent bone cells (90-95\%), while osteoblasts are estimated to constitute $4-6 \%$ and osteoclasts around 1-2\%. Other cell sources may be lining cells, MSCs, or even adipocytes.

At the intersection of hOB and hOC profiles, 101 miRNAs shared between both cell types were identified, accounting for about $30-40 \%$ of miRNAs detected in these cells. Moreover, seven miRNAs detected in both cell types were not detected in bone tissue samples (miR-1228-5p, miR-193b-5p, miR-197-3p, miR-3127-3p, miR-320d, miR-324-3p and miR-455-3p).

miRNAs from bone tissue profile were then sorted by expression level. Of the most highly expressed bone-related miRNAs, $13(65 \%)$ of the top 20 and $9(90 \%)$ of the top 10 were found in osteoblasts and/or osteoclasts. Table 2 shows the top 10 most highly expressed miRNAs in bone and their localization.

A total of 266 miRNAs were detected in osteoblasts, of which $243(91 \%)$ were also present in total bone samples, representing $38 \%$ of all 641 bone miRNAs. Of these 243 miRNAs, 94 were also expressed in osteoclasts. miRNAs present in all osteoblast samples were sorted by expression level. Of the most highly expressed human miRNAs, $75 \%$ of the top 20 were found in bone tissue and the other $25 \%$ were detected only in the osteoblast profile (miR-1282, hsamiR-1228-5p, miR-508-3p, miR-3127-3p and miR-1825). The top 10 most highly expressed miRNAs in hOBs are shown in Table 3.

In osteoclasts, 340 miRNAs were detected, of which 196 (58\%) were also present in the bone tissue profile, representing $31 \%$ of all miRNAs detected in total bone tissue. miRNAs present in all osteoclast samples were sorted by expression level. In this case, all 20 of the most highly expressed osteoclast-related miRNAs were found in bone tissue. Table 4 shows the top 10 most highly expressed miRNAs in hOCs.

As a sensitivity analysis, the same intersection analysis was performed considering only the miRNAs detected in all samples. A slight decrease in the number of miRNAs was detected in most of the sample groups but the proportions remained the same (Supplemental figure 3). 


\section{Discussion}

Three microarrays were performed; one from whole bone tissue, one from cultured osteoblasts and one from differentiated osteoclasts, with the aim of getting a broad overview of microRNA expression in bone tissue.

To reduce interindividual variability, only miRNAs present in at least $2 / 4$ of the hOB samples, $2 / 5$ of the hOC samples, and 3/6 of the bone samples were included in the intersection analysis. Many miRNAs were detected exclusively in one individual; for instance, 44 miRNAs were detected in a single osteoblast sample. It is obvious that great interindividual variability exists among patients and must be taken into account when using human samples.

Unlike the osteoblast profile, in which the vast majority of miRNAs were also observed in the bone array, only half of miRNAs detected in osteoclasts were also detected in total bone tissue. One possible explanation is the low percentage of osteoclasts in bone, which makes it difficult to detect osteoclast-specific miRNAs when total bone tissue is analyzed. Hence, only miRNAs highly expressed in osteoclasts can be detected in the bone tissue array. Another explanation may be the methodology used to obtain osteoclast cells, since they were differentiated from blood mononuclear cells in vitro instead of isolating them in-situ from bone tissue. Remarkably, all top 20 most highly expressed osteoclast-related miRNAs were found in bone tissue. This fact corroborates the low osteoclast presence hypothesis: only highly expressed miRNAs in osteoclasts are detected in the bone tissue array, which is a limitation of whole tissue studies.

On the other hand, the detection of some true miRNAs expressed in human osteoclasts might be lost due to the in vitro procedure, and we cannot rule out that some miRNAs detected in hOCs may be artifacts of the artificial laboratory cell differentiation, which would explain their absence in the bone array. Unfortunately, due to the relatively small number of osteoclasts in human bone tissues, in vitro differentiation from peripheral blood mononuclear cells is the most common current methodology used to obtain hOCs.

In this regard, seven miRNAs were detected in both hOB and hOC cells but not in bone tissue samples. These miRNAs might be attributable to some aspect related to the in vitro culture conditions. Moreover, of the top 20 most highly expressed miRNAs in hOB, $25 \%$ were detected 
only in the osteoblast profile, suggesting that the in vitro conditions of cultured cells create an artificial environment leading to an altered miRNA expression. On the other hand, hOB cultures were obtained from knee bone whereas total bone samples were obtained from femoral neck and therefore, we cannot rule out some site-specific differences of miRNA expression profiling between knee and femoral trabecular bones.

Focusing on the top 10 most highly expressed miRNAs in bone; some of them have been previously linked to bone in the literature (see Table 2). Some reports have described their association with osteosarcoma: ectopic expression of miR-26b in the human U2OS cell line inhibits proliferation, migration, invasion, cell cycle arrest, and induction of apoptosis [9]; miR20a down-regulates Fas expression in osteosarcoma, thus contributing to the metastatic potential of osteosarcoma cells [10]. Also, miR-20a has been predicted to target key genes related to osteogenic differentiation, such as BMP2 and RUNX2 [11]. In addition, miR27a expression was found to be significantly reduced in osteoporotic patients [12]. Interestingly, several reports have focused on the regulatory functions of miR-223 in bone metabolism. Its expression has been reported to play a negative role in the differentiation of both osteoblast and osteoclast [13].

Two of the most highly expressed miRNAs in osteoblasts have been recently involved in the osteoporosis phenotype. In this regard, two SNPs -rs6430498 in the miR-3679 and rs12512664 in the miR-4274- were significantly associated with femoral neck bone mineral density and both miRNAs were found overexpressed in fractured bone [14].

In our analysis, miR-146a-5p, miR-378a-3p and miR-342-3p were 3 of the 20 most highly expressed miRNAs in mature osteoclasts. These miRNAs were detected as significantly upregulated during osteoclast differentiation by de la Rica et al.[15]. In their study, osteoclasts were also differentiated from primary monocytes obtained from three human donors, and mature osteoclasts were obtained 21 days after RANKL/M-CSF stimulation. Further studies are needed to fully explore the involvement of these miRNAs in osteoclast regulation.

As observed during our study and described in the literature, the vast majority of miRNAs are non-restrictive to an exclusive cell type. Thus, a broad view of miRNA function in any one tissue would be a fundamental prerequisite to understand the complete regulation mechanism of 
miRNAs in the tissue, which in turn would contribute to the improvement of miRNA-based therapies in that specific tissue.

Primary osteoblasts and osteoclasts are the most common bone cells used in research because of the availability of cell isolation protocols. One limitation of the present study is the lack of profiling for osteocytes, the most common bone cells. Although several techniques have been published for the isolation of osteocytes from mouse bone, no such technique has yet been established for human osteocytes. Recently, Prideaux et al. [16] developed a protocol for the isolation of osteocytes from human trabecular bone samples obtained during surgery. The next step would be to obtain the miRNA signature of the osteocytes, in order to provide an even more comprehensive view of the miRNA signature in human bone tissue. Other limitation is that bone samples were obtained from osteoarthritic joints and some bone abnormalities may be present. Due to ethical reasons the collection of joint samples of healthy individuals is not allowed. However, in an attempt to minimize this potential caveat, we were careful in obtaining bone samples from a location as far away as possible from the osteoarthritic lesion.

Overall, this study presents a broad view of miRNAs expressed in bone tissue that will contribute to enhancing our knowledge about the miRNA field in human bone. This study also provides information for further studies using bone-related miRNAs and for designing future miRNA-based therapies.

\section{Conflict of interests}

The authors declare that they have no conflict of interest.

\section{Acknowledgments}

The authors thank Elaine M. Lilly, PhD, for helpful advice and critical reading of the manuscript. Eulàlia Puigdecanet, $\mathrm{PhD}$, is also appreciated for her help with the arrays. This work was supported by the Red Temática de Investigación Cooperativa en Envejecimiento y Fragilidad (RETICEF; RD12/0043/0022) and Centro de Investigación Biomédica en Red de Fragilidad y Envejecimiento Saludable (CIBERFES; CB16/10/00245), and the Grants PI10/01537, PI13/00116 and PI13/00444 from FIS (Carlos III Health Institute, Science and Innovation 
Ministry); SAF2016-75948-R, from Ministerio de Economia y Competitividad, and 2014SGR-932 from Generalitat de Catalunya. FEDER funds also supported this study.

\section{References}

1. Chambers TJ. Regulation of the differentiation and function of osteoclasts. J Pathol. 2000;192(1):4-13. doi:10.1002/1096-9896(2000).

2. Tatsumi S, Ishii K, Amizuka N, Li M, Kobayashi T, Kohno K et al. Targeted ablation of osteocytes induces osteoporosis with defective mechanotransduction. Cell Metab. 2007;5(6):464-75. doi:10.1016/j.cmet.2007.05.001.

3. Bonewald LF, Johnson ML. Osteocytes, mechanosensing and Wnt signaling. Bone. 2008;42(4):606-15. doi:10.1016/j.bone.2007.12.224.

4. Jing D, Hao J, Shen Y, Tang G, Li ML, Huang SH et al. The role of microRNAs in bone remodeling. Int J Oral Sci. 2015;7(3):131-43. doi:10.1038/ijos.2015.22.

5. De-Ugarte L, Yoskovitz G, Balcells S, Guerri-Fernandez R, Martinez-Diaz S, Mellibovsky L et al. MiRNA profiling of whole trabecular bone: identification of osteoporosis-related changes in MiRNAs in human hip bones. BMC Med Genomics. 2015;8(1):75. doi:10.1186/s12920-0150149-210.1186/s12920-015-0149-2 [pii].

6. Garmilla-Ezquerra P, Sanudo C, Delgado-Calle J, Perez-Nunez MI, Sumillera M, Riancho JA. Analysis of the Bone MicroRNome in Osteoporotic Fractures. Calcif Tissue Int. 2014. doi:10.1007/s00223-014-9935-7.

7. Panach L, Mifsut D, Tarin JJ, Cano A, Garcia-Perez MA. Serum Circulating MicroRNAs as Biomarkers of Osteoporotic Fracture. Calcif Tissue Int. 2015;97(5):495-505. doi:10.1007/s00223-015-0036-z10.1007/s00223-015-0036-z [pii].

8. Seeliger C, Karpinski K, Haug A, Vester H, Schmitt A, Bauer J et al. Five Freely Circulating miRNAs and Bone Tissue miRNAs are Associated with Osteoporotic Fractures. J Bone Miner Res. 2014. doi:10.1002/jbmr.2175.

9. Du JY, Wang LF, Wang Q, Yu LD. miR-26b inhibits proliferation, migration, invasion and apoptosis induction via the downregulation of 6-phosphofructo-2-kinase/fructose-2,6bisphosphatase-3 driven glycolysis in osteosarcoma cells. Oncol Rep. 2015;33(4):1890-8. doi:10.3892/or.2015.3797.

10. Huang G, Nishimoto K, Zhou Z, Hughes D, Kleinerman ES. miR-20a encoded by the miR17-92 cluster increases the metastatic potential of osteosarcoma cells by regulating Fas expression. Cancer Res. 2012;72(4):908-16. doi:10.1158/0008-5472.CAN-11-1460. 
11. Yu F, Cui $Y$, Zhou $X$, Zhang $X$, Han J. Osteogenic differentiation of human ligament fibroblasts induced by conditioned medium of osteoclast-like cells. Biosci Trends. 2011;5(2):4651. doi:414 [pii].

12. You L, Pan L, Chen L, Gu W, Chen J. MiR-27a is Essential for the Shift from Osteogenic Differentiation to Adipogenic Differentiation of Mesenchymal Stem Cells in Postmenopausal Osteoporosis. Cell Physiol Biochem. 2016;39(1):253-65. doi:10.1159/000445621.

13. Xie Y, Zhang L, Gao Y, Ge W, Tang P. The Multiple Roles of Microrna-223 in Regulating Bone Metabolism. Molecules. 2015;20(10):19433-48. doi:10.3390/molecules201019433.

14. De-Ugarte L, Caro-Molina E, Rodriguez-Sanz M, Garcia-Perez MA, Olmos JM, SosaHenriquez $M$ et al. SNPs in bone-related miRNAs are associated with the osteoporotic phenotype. Scientific Reports. 2017;7(1):516. doi:10.1038/s41598-017-00641-7.

15. de la Rica L, Garcia-Gomez A, Comet NR, Rodriguez-Ubreva J, Ciudad L, Vento-Tormo R et al. NF-kappaB-direct activation of microRNAs with repressive effects on monocyte-specific genes is critical for osteoclast differentiation. Genome Biol. 2015;16:2. doi:10.1186/s13059-0140561-5.

16. Prideaux M, Schutz C, Wijenayaka AR, Findlay DM, Campbell DG, Solomon LB et al. Isolation of osteocytes from human trabecular bone. Bone. 2016;88:64-72. doi:10.1016/j.bone.2016.04.017.

17. Chen X, Xiao W, Chen W, Liu X, Wu M, Bo Q et al. MicroRNA-26a and -26b inhibit lens fibrosis and cataract by negatively regulating Jagged-1/Notch signaling pathway. Cell Death Differ. 2017. doi:10.1038/cdd.2016.152.

18. Li Y, Fan L, Liu S, Liu W, Zhang H, Zhou $T$ et al. The promotion of bone regeneration through positive regulation of angiogenic-osteogenic coupling using microRNA-26a. Biomaterials.2013;34(21):504858.doi:10.1016/j.biomaterials.2013.03.052S01429612(13)003621 [pii].

19. Zhang JF, Fu WM, He ML, Xie WD, Lv Q, Wan G et al. MiRNA-20a promotes osteogenic differentiation of human mesenchymal stem cells by co-regulating BMP signaling. RNA Biol. 2011;8(5):829-38. doi:10.4161/rna.8.5.16043.

20. Poliseno L, Pitto L, Simili M, Mariani L, Riccardi L, Ciucci A et al. The proto-oncogene LRF is under post-transcriptional control of MiR-20a: implications for senescence. PLoS One. 2008;3(7):e2542. doi:10.1371/journal.pone.0002542.

21. Zhan $\mathrm{XH}, \mathrm{Xu} \mathrm{QY}$, Tian R, Yan $\mathrm{H}$, Zhang $\mathrm{M}, \mathrm{Wu} \mathrm{J}$ et al. MicroRNA16 regulates glioma cell proliferation, apoptosis and invasion by targeting Wip1-ATM-p53 feedback loop. Oncotarget. 2017. doi:10.18632/oncotarget.18510. 
22. Zhao D, Zhang Y, Song L. MiR-16-1 Targeted Silences Far Upstream Element Binding Protein 1 to Advance the Chemosensitivity to Adriamycin in Gastric Cancer. Pathol Oncol Res. 2017. doi:10.1007/s12253-017-0263-x.

23. Zhang W, Zhou J, Zhu X, Yuan H. MiR-126 reverses drug resistance to TRAlL through inhibiting the expression of C-FLIP in cervical cancer. Gene. 2017. doi:10.1016/j.gene.2017.06.055.

24. Hao XZ, Fan HM. Identification of miRNAs as atherosclerosis biomarkers and functional role of miR-126 in atherosclerosis progression through MAPK signalling pathway. Eur Rev Med Pharmacol Sci. 2017;21(11):2725-33.

25. Tamaddon G, Geramizadeh B, Karimi MH, Mowla SJ, Abroun S. miR-4284 and miR-4484 as Putative Biomarkers for Diffuse Large B-Cell Lymphoma. Iran J Med Sci. 2016;41(4):334-9.

26. Koukos G, Polytarchou C, Kaplan JL, Oikonomopoulos A, Ziring D, Hommes DW et al. A microRNA signature in pediatric ulcerative colitis: deregulation of the miR-4284/CXCL5 pathway in the intestinal epithelium. Inflamm Bowel Dis. 2015;21(5):996-1005. doi:10.1097/MIB.0000000000000339.

27. McDaniel K, Huang L, Sato K, Wu N, Annable T, Zhou T et al. The let-7/Lin28 axis regulates activation of hepatic stellate cells in alcoholic liver injury. J Biol Chem. 2017;292(27):11336-47. doi:10.1074/jbc.M116.773291.

28. Hu XP, Xie Q, Chen CF, Zhang W, Yu B. Let-7a Inhibits T-Cell Proliferation and IFN-gamma Secretion by Down-Regulating STAT3 Expression in Patients with Psoriasis. Cell Physiol Biochem. 2017;42(1):115-25. doi:10.1159/000477120.

29. Guo D, Li Q, Lv Q, Wei Q, Cao S, Gu J. MiR-27a targets sFRP1 in hFOB cells to regulate proliferation, apoptosis and differentiation. PLoSOne.2014;9(3):e91354. doi:10.1371/journal.pone.0091354.

30. Wang $T, X u Z$ Z. miR-27 promotes osteoblast differentiation by modulating Wnt signaling. Biochem Biophys Res Commun. 2010;402(2):186-9. doi:10.1016/j.bbrc.2010.08.031.

31. Huang HC, Yu HR, Hsu TY, Chen IL, Huang HC, Chang JC et al. MicroRNA-142-3p and let$7 g$ Negatively Regulates Augmented IL-6 Production in Neonatal Polymorphonuclear Leukocytes. Int J Biol Sci. 2017;13(6):690-700. doi:10.7150/ijbs.17030.

32. Liu JM, Long $X H$, Zhang GM, Zhou $Y$, Chen $X Y$, Huang SH et al. Let-7g reverses malignant phenotype of osteosarcoma cells by targeting Aurora-B. Int J Clin Exp Pathol. 2014;7(8):4596606. 
33. Ji J, Zhao L, Budhu A, Forgues M, Jia HL, Qin LX et al. Let-7g targets collagen type I alpha2 and inhibits cell migration in hepatocellular carcinoma. J Hepatol. 2010;52(5):690-7. doi:10.1016/j.jhep.2009.12.025.

34. Das DK, Naidoo M, Ilboudo A, Park JY, Ali T, Krampis K et al. miR-1207-3p regulates the androgen receptor in prostate cancer via FNDC1/fibronectin. Exp Cell Res. 2016;348(2):190200. doi:10.1016/j.yexcr.2016.09.021.

35. Arora S, Saini S, Fukuhara S, Majid S, Shahryari V, Yamamura S et al. MicroRNA-4723 inhibits prostate cancer growth through inactivation of the Abelson family of nonreceptor protein tyrosine kinases. PLoS One. 2013;8(11):e78023. doi:10.1371/journal.pone.0078023.

36. Kalantari P, Harandi OF, Agarwal S, Rus F, Kurt-Jones EA, Fitzgerald KA et al. miR-718 represses proinflammatory cytokine production through targeting phosphatase and tensin homolog (PTEN). J Biol Chem. 2017;292(14):5634-44. doi:10.1074/jbc.M116.749325.

37. Wang ZD, Qu FY, Chen YY, Ran ZS, Liu HY, Zhang HD. Involvement of microRNA-718, a new regulator of EGR3, in regulation of malignant phenotype of HCC cells. J Zhejiang Univ Sci B. 2017;18(1):27-36. doi:10.1631/jzus.B1600205.

38. Jurjevic I, Miyajima M, Ogino I, Akiba C, Nakajima M, Kondo A et al. Decreased Expression of hsa-miR-4274 in Cerebrospinal Fluid of Normal Pressure Hydrocephalus Mimics with Parkinsonian Syndromes. J Alzheimers Dis. 2017;56(1):317-25. doi:10.3233/JAD-160848.

39. Ye Y, Wei Y, Xu Y, Li Y, Wang R, Chen J et al. Induced MiR-1249 expression by aberrant activation of Hedegehog signaling pathway in hepatocellular carcinoma. Exp Cell Res. 2017;355(1):9-17. doi:10.1016/j.yexcr.2017.03.010.

40. Lam CS, Ng L, Chow AK, Wan TM, Yau S, Cheng NS et al. Identification of microRNA 885$5 p$ as a novel regulator of tumor metastasis by targeting CPEB2 in colorectal cancer. Oncotarget. 2017;8(16):26858-70. doi:10.18632/oncotarget.15844.

41. Zhang Z, Yin J, Yang J, Shen W, Zhang C, Mou W et al. miR-885-5p suppresses hepatocellular carcinoma metastasis and inhibits Wnt/beta-catenin signaling pathway. Oncotarget. 2016;7(46):75038-51. doi:10.18632/oncotarget.12602.

42. Meier C, Hardtstock P, Joost S, Alla V, Putzer BM. p73 and IGF1R Regulate Emergence of Aggressive Cancer Stem-like Features via miR-885-5p Control. Cancer Res. 2016;76(2):197205. doi:10.1158/0008-5472.CAN-15-1228.

43. Wang F, Zhang H, Xu N, Huang N, Tian C, Ye A et al. A novel hypoxia-induced miR-147a regulates cell proliferation through a positive feedback loop of stabilizing HIF-1alpha. Cancer Biol Ther. 2016;17(8):790-8. doi:10.1080/15384047.2016.1195040. 
44. Niu X, Fu N, Du J, Wang R, Wang Y, Zhao S et al. miR-1273g-3p modulates activation and apoptosis of hepatic stellate cells by directly targeting PTEN in HCV-related liver fibrosis. FEBS Lett. 2016;590(16):2709-24. doi:10.1002/1873-3468.12309.

45. Guo J, Sang Y, Yin T, Wang B, Yang W, Li X et al. miR-1273g-3p participates in acute glucose fluctuation-induced autophagy, dysfunction, and proliferation attenuation in human umbilical vein endothelial cells. Am J Physiol Endocrinol Metab. 2016;310(9):E734-43. doi:10.1152/ajpendo.00444.2015.

46. Yu F, Fan X, Chen B, Dong P, Zheng J. Activation of Hepatic Stellate Cells is Inhibited by microRNA-378a-3p via Wnt10a. Cell Physiol Biochem. 2016;39(6):2409-20. doi:10.1159/000452509.

47. Yu F, Yang J, Huang K, Pan X, Chen B, Dong $P$ et al. The Epigenetically-Regulated microRNA-378a Targets TGF-beta2 in TGF-beta1-Treated Hepatic Stellate Cells. Cell Physiol Biochem. 2016;40(1-2):183-94. doi:10.1159/000452536.

48. Gao F, Xiong X, Pan W, Yang X, Zhou C, Yuan Q et al. A Regulatory MDM4 Genetic Variant Locating in the Binding Sequence of Multiple MicroRNAs Contributes to Susceptibility of Small Cell Lung Cancer. PLoS One. 2015;10(8):e0135647. doi:10.1371/journal.pone.0135647.

49. Hu R, Liu W, Li H, Yang L, Chen C, Xia ZY et al. A Runx2/miR-3960/miR-2861 regulatory feedback loop during mouse osteoblast differentiation. J Biol Chem. 2011;286(14):12328-39. doi:10.1074/jbc.M110.176099.

50. Xin J, Li J, Feng Y, Wang L, Zhang Y, Yang R. Downregulation of long noncoding RNA HOTAIRM1 promotes monocyte/dendritic cell differentiation through competitively binding to endogenous miR-3960. Onco Targets Ther. 2017;10:1307-15. doi:10.2147/OTT.S124201.

51. Li Q, Wang N, Wei H, Li C, Wu J, Yang G. miR-24-3p Regulates Progression of Gastric Mucosal Lesions and Suppresses Proliferation and Invasiveness of N87 Via Peroxiredoxin 6. Dig Dis Sci. 2016;61(12):3486-97. doi:10.1007/s10620-016-4309-9.

52. Ye SB, Zhang H, Cai TT, Liu YN, Ni JJ, He J et al. Exosomal miR-24-3p impedes T-cell function by targeting FGF11 and serves as a potential prognostic biomarker for nasopharyngeal carcinoma. J Pathol. 2016;240(3):329-40. doi:10.1002/path.4781.

53. Yin Y, Zhong J, Li SW, Li JZ, Zhou M, Chen Y et al. TRIM11, a direct target of miR-24-3p, promotes cell proliferation and inhibits apoptosis in colon cancer. Oncotarget. 2016;7(52):86755-65. doi:10.18632/oncotarget.13550.

54. Yu G, Jia Z, Dou Z. miR-24-3p regulates bladder cancer cell proliferation, migration, invasion and autophagy by targeting DEDD. Oncol Rep. 2017;37(2):1123-31. doi:10.3892/or.2016.5326. 
55. Yuan Y, Kluiver J, Koerts J, de Jong D, Rutgers B, Abdul Razak FR et al. miR-24-3p Is Overexpressed in Hodgkin Lymphoma and Protects Hodgkin and Reed-Sternberg Cells from Apoptosis. Am J Pathol. 2017;187(6):1343-55. doi:10.1016/j.ajpath.2017.02.016.

56. Kang L, Yang C, Song Y, Liu W, Wang K, Li S et al. MicroRNA-23a-3p promotes the development of osteoarthritis by directly targeting SMAD3 in chondrocytes. Biochem Biophys Res Commun. 2016;478(1):467-73. doi:10.1016/j.bbrc.2016.06.071.

57. Black JC, Zhang H, Kim J, Getz G, Whetstine JR. Regulation of Transient Site-specific Copy Gain by MicroRNA. J Biol Chem. 2016;291(10):4862-71. doi:10.1074/jbc.M115.711648.

58. Ryan B, Logan BJ, Abraham WC, Williams JM. MicroRNAs, miR-23a-3p and miR-151-3p, Are Regulated in Dentate Gyrus Neuropil following Induction of Long-Term Potentiation In Vivo. PLoS One. 2017;12(1):e0170407. doi:10.1371/journal.pone.0170407.

59. Xie X, Liu H, Wang M, Ding F, Xiao H, Hu F et al. miR-342-3p targets RAP2B to suppress proliferation and invasion of non-small cell lung cancer cells. Tumour Biol. 2015;36(7):5031-8. doi:10.1007/s13277-015-3154-3.

60. Zhang S, Liu L, Lv Z, Li Q, Gong W, Wu H. MicroRNA-342-3p Inhibits the Proliferation, Migration, and Invasion of Osteosarcoma Cells By Targeting Astrocyte-Elevated Gene-1 (AEG1). Oncol Res. 2017. doi:10.3727/096504017X14886485417426.

61. Li XR, Chu HJ, Lv T, Wang L, Kong SF, Dai SZ. miR-342-3p suppresses proliferation, migration and invasion by targeting FOXM1 in human cervical cancer. FEBS Lett. 2014;588(17):3298-307. doi:10.1016/j.febslet.2014.07.020.

62. Mody HR, Hung SW, AlSaggar M, Griffin J, Govindarajan R. Inhibition of SAdenosylmethionine-Dependent Methyltransferase Attenuates TGFbeta1-Induced EMT and Metastasis in Pancreatic Cancer: Putative Roles of miR-663a and miR-4787-5p. Mol Cancer Res. 2016;14(11):1124-35. doi:10.1158/1541-7786.MCR-16-0083. 
Table 1. Patients' characteristics, by sample group

\begin{tabular}{|c|c|c|c|}
\hline $\begin{array}{c}\text { Patients' } \\
\text { characteristics }\end{array}$ & $\begin{array}{c}\text { Whole bone tissue } \\
\mathbf{n}=\mathbf{6}\end{array}$ & $\begin{array}{c}\text { Osteoblasts } \\
\mathbf{n}=\mathbf{4}\end{array}$ & $\begin{array}{c}\text { Osteoclasts } \\
\mathbf{n}=\mathbf{5}\end{array}$ \\
\hline Age (years) & $72.5 \pm 7.42$ & $71.5 \pm 9.94$ & $65.4 \pm 5.08$ \\
BMI $\left(\mathbf{k g} / \mathbf{m}^{2}\right)$ & $26.06 \pm 3.25$ & $30.4 \pm 2.43$ & $22.85 \pm 2.56$ \\
LS BMD $\left(\mathbf{g} / \mathbf{c m}^{2}\right)$ & $1.03 \pm 0.18$ & $0.97 \pm 0.41$ & $0.80 \pm 0.13$ \\
FN BMD $\left(\mathbf{g} / \mathbf{c m}^{2}\right)$ & $0.794 \pm 0.07$ & $0.71 \pm 0.11$ & $0.65 \pm 0.08$ \\
Sample source & Femoral neck & Knee & Blood \\
\hline
\end{tabular}

BMI, body mass index; LS BMD, lumbar spine bone mineral density; FN BMD, femoral neck bone mineral density

Table 2. Top ten most expressed miRNAs in the bone tissue array (listed in order of expression levels) and their localization. Validated and predicted target genes are described.

\begin{tabular}{|c|c|c|c|}
\hline miRNA & Localization & Target gene(s) & References \\
\hline $\operatorname{miR}-26 b-5 p$ & $\mathrm{OB}$ & Jagged-1, FUT4 & [17], [18] \\
\hline miR-20a-5p & $\mathrm{OC}$ & $\begin{array}{l}\text { BMP2, RUNX2, PPARY, Bambi, } \\
\text { Crim1, LRF }\end{array}$ & [10], [19], [20] \\
\hline miR-16-5p & $\mathrm{OB} \& \mathrm{OC}$ & Wip1, FUBP1 & [21], [22] \\
\hline miR-126-3p & $\mathrm{OC}$ & $C-F L I P, M A P 3 K 10$ & [23], [24] \\
\hline miR-4284 & OB \& OC & $\begin{array}{l}\text { TRAF4, BCL 10, HDAC, } \\
\text { HOXA1, PTEN, CXCL5 }\end{array}$ & [25], [26] \\
\hline let-7a-5p & $\mathrm{OB} \& \mathrm{OC}$ & Lin28B, HMGA2, STAT3 & [27], [28] \\
\hline miR-223-3p & ONLY BONE & $\begin{array}{l}\text { C/EBP } \beta, I K K \alpha, N F I A, \\
\text { FGFR2 }\end{array}$ & [13] \\
\hline miR-4791 & OB & Unknown & \\
\hline miR-27a-3p & $\mathrm{OC}$ & $s F R P 1, A P C$, Mef2c & [29], [30], [12] \\
\hline let-7g-5p & $\mathrm{OB} \& \mathrm{OC}$ & IL-6, Aurora-B, COL1A2 & [31], [32], [33] \\
\hline
\end{tabular}

OB, osteoblasts; OC, osteoclasts 
Table 3. Top ten most expressed miRNAs in the hOB array (listed in order of expression levels) and the overlap in total bone tissue and hOC arrays.

Validated and predicted target genes are described.

\begin{tabular}{|c|c|c|c|}
\hline miRNA & Overlapping & Target gene(s) & References \\
\hline miR-3679-3p & BONE & Unknown & \\
\hline miR-1207-3p & BONE & FNDC1 & [34] \\
\hline miR-4723-3p & BONE & $A b / 1, A b / 2$ & [35] \\
\hline miR-718 & BONE & PTEN, EGR3 & [36], [37] \\
\hline miR-4274 & BONE & SLC18A2 & [38] \\
\hline miR-1249 & BONE & PTCH1 & [39] \\
\hline miR-1282 & ONLY OB & Unknown & \\
\hline miR-3614-3p & BONE & Unknown & \\
\hline miR-885-5p & BONE \& OC & $\begin{array}{l}\text { CPEB2, } \\
\text { CTNNB1, IGF1R }\end{array}$ & [40], [41], [42] \\
\hline $\mathrm{miR}-147 a$ & BONE & $H I F-3 \alpha$ & [43] \\
\hline
\end{tabular}

OB, osteoblasts; OC, osteoclasts

Table 4. Top ten most expressed miRNAs in the hOC array (listed in order of expression levels) and the overlap in total bone tissue and hOB arrays. Validated and predicted target genes are described.

\begin{tabular}{|c|c|c|c|}
\hline miRNA & Overlapping & Target gene(s) & References \\
\hline miR-1273g-3p & BONE \& OB & PTEN, CNR1 & [44], [45] \\
\hline miR-378a-3p & BONE & TGF-62, Wnt10a & [46], [47] \\
\hline miR-4497 & BONE \& OB & Unknown & \\
\hline miR-191-5p & BONE \& OB & MDM4 & [48] \\
\hline miR-3960 & BONE \& OB & Hoxa2, HOXA1 & [49], [50] \\
\hline miR-3665 & BONE & Unknown & \\
\hline miR-24-3p & BONE \& OB & $\begin{array}{l}\text { Prdx-6, FGF11, TRIM11, DEDD, } \\
\text { CDKN1B/P27kip1, MYC }\end{array}$ & $\begin{array}{c}\text { [51], [52], } \\
{[53],[54],} \\
{[55]}\end{array}$ \\
\hline $\operatorname{miR}-23 a-3 p$ & BONE \& OB & $\begin{array}{l}\text { SMAD3, KDM4A, BCLAF1, ABT1, } \\
\text { RUNX1T1, CTCF, GZF1, GAZF1, } \\
\text { ZC3H8, ZFR, ZBTB38 }\end{array}$ & $\begin{array}{c}{[56],[57],} \\
{[58]}\end{array}$ \\
\hline miR-342-3p & BONE & $R A P 2 B, A E G-1, F O X M 1$ & $\begin{array}{c}59],[60], \\
{[61]}\end{array}$ \\
\hline $\operatorname{miR}-4787-5 p$ & BONE \& OB & TGFB1 & [62] \\
\hline
\end{tabular}

$\mathrm{OB}$, osteoblasts 


\section{Figure legends}

Fig. 1 Global expression analysis of bone-related miRNAs. Venn diagram showing data obtained from the microarray analysis of miRNAs detected in whole bone samples, primary osteoblasts and osteoclasts 


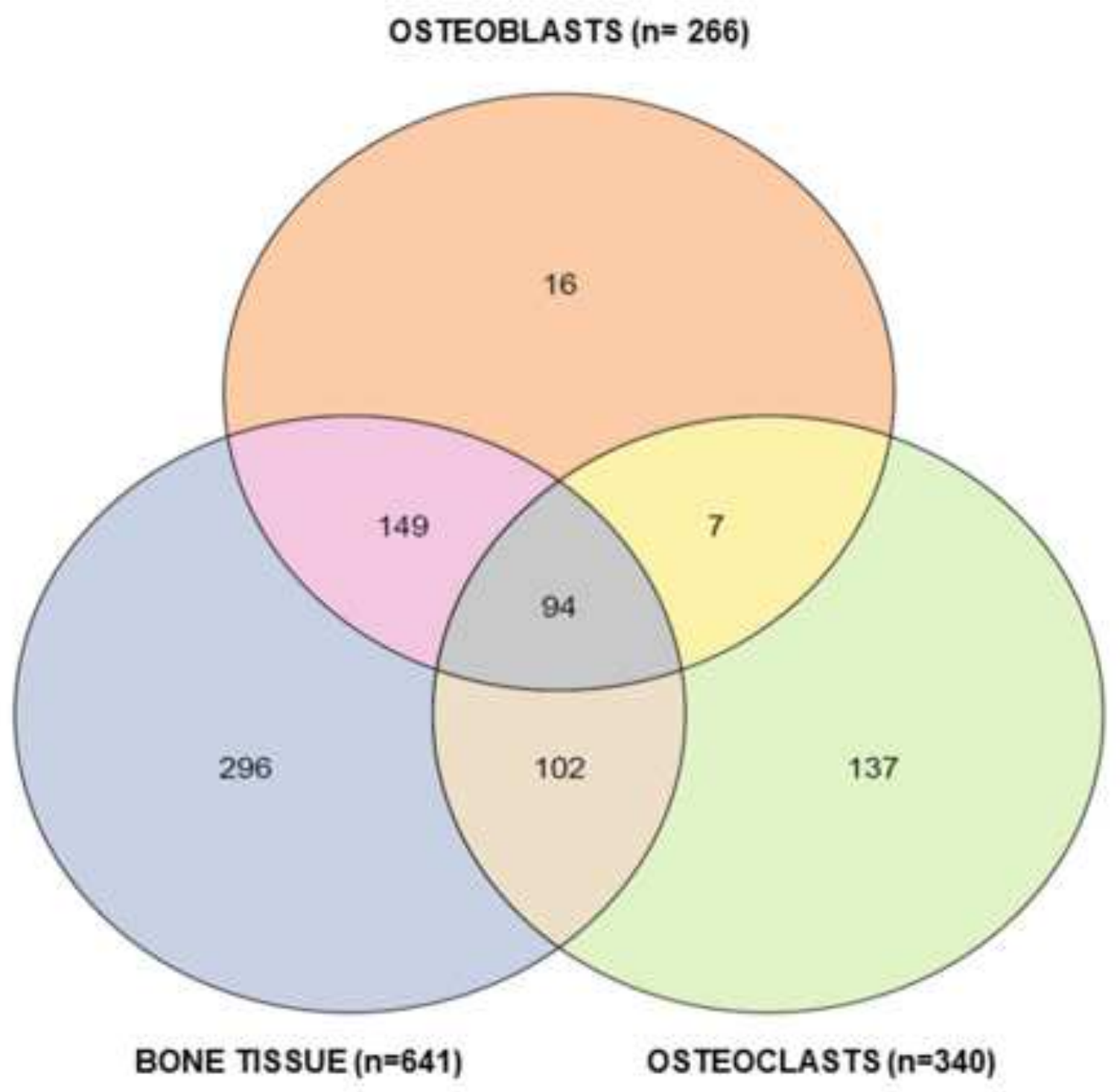


Click here to access/download Supplementary Material Supplemental fig 1.TIF 
Click here to access/download Supplementary Material Supplemental fig 2.TIF 
Click here to access/download Supplementary Material Supplemental fig 3.tif 
Click here to access/download Certification Form Certification form.pdf 